\title{
A New Concept to Detect Isomorphism in Kinematic Chains using Fuzzy Similarity Index
}

\author{
Syed Shane Haider Rizvi \\ Research Scholar \\ Mech Engg Department \\ J.M.I. New Delhi-25
}

\author{
Ali Hasan, Ph.D \\ Assistant Professor \\ Mech Engg Department \\ J.M.I. New Delhi-25
}

\author{
R.A Khan \\ Professor \\ Mech Engg Department \\ Galgotia Univ.G.Noida
}

\begin{abstract}
This paper presents a new method based on fuzzy similarity measure to investigate isomorphism among kinematic chains having same number of links and degrees of freedom (d.o.f.). Two different similarity measures which are available in the literature are used to compare the numerous distinct chains.
\end{abstract}

\section{General Terms}

Isomorphism detection

\section{Keywords}

Fuzzy similarity measure, kinematic chain, isomorphism

\section{INTRODUCTION}

One of the most encountered problems in the structural synthesis of kinematic chains is to check the isomorphism between the two chains. The two kinematic chains are isomorphic, only if there is one to one correspondence between the links of the two chains.

The detection of isomorphism among two kinematic chains with same number of links is necessary to prevent duplication and omission of a chain which is mechanically more useful than its isomer. A lot of literature related to isomorphism detection is available but still there is a scope for an efficient, simple and reliable method and this paper is an attempt in this direction. Visual methods [1] were only suitable for kinematic chains with a small number of links. Characteristic polynomial method [2] which requires lengthy calculations and later counter examples were also reported [3]. Rao and Raju presented a method of loops for multi degree of freedom chains [4].Characteristic polynomial of structural matrix was proposed by Yan and Hwang [5]; but the method is uneconomical because of more computational time. Mruthyunjaya proposed a method of binary coding for structural synthesis of kinematic chains [6]. Agarwal and Rao proposed Variable permanent function to identify multi loop kinematic chains [7]. Method of Canonical coding of kinematic chains is presented by Ambekar and Agarwal a [8] but it is computationally uneconomical for large kinematic chain. Hamming number technique [9] is reliable and efficient, but when the primary Hamming string fails, it require the computation of the secondary Hamming string which is time consuming. Shin and Krishna Murthy presents some rules for relabeling its vertices canonically for a given kinematic chain [10]. However where a higher number of symmetry group elements in the kinematic chain are present. it becomes computationally inefficient The degree code [11] generated by the contracted link adjacency matrix of a chain was also proposed for testing the isomorphism. Yadav and Pratap present a method of link distance for the detection of isomorphism [12]. A method based on artificial neural network theory by Kong et al. was presented [13]. Quist and Soni give a loop method for kinematic chains [14]. A new method based on eigenvalues and eigenvectors of adjacent matrices of chains was also proposed [15]. The reliability of the existing spectral techniques for isomorphism detection was challenged by Sunkari, R.P., and Schmidt [16]. Huafeng Ding and Zhen Huang [17] shows that the characteristic polynomial and eigen value approach fails and proposed a method based on the perimeter topological graph and give rules for relabeling its vertices canonically. Hasan and Khan [18] presented a method based on degrees of freedom of kinematic pairs. All the above methods developed so far uses the graphs of the Kinematic chain and their adjacency matrices in one or the other way.

\section{THEORY}

A kinematic chain can be represented by a graph in which the links become vertices or nodes and the joints become edges if there is an edge between two vertices, they are considered to be "adjacent". The "degree" of a vertex is the number of vertices adjacent to it and a graph is termed as "connected" if there is some path between every pair of vertices in the graph. A graph is "complete" if all pairs of vertices are adjacent i.e. All nodes are connected to every other node. The kinematic chain corresponding to a complete graph will have a negative degree of freedom and it is termed as a redundant structure not a kinematic chain because of its immobility, for a kinematic chain it is not possible to be represented by a complete or fully connected graph hence a membership grade to every node of the actual chain graph on a scale of ' 0 ' to ' 1 ' is assigned, where ' 0 ' represents that the node is not connected to any other node of the graph while ' 1 ' represents that the node is connected to every other node of the chain graph hence for the actual chain graph both the crisp numbers does not exists hence a kinematic chain can be represented by membership grade between 0 and 1[19]which represents that up to what extent the chain is comparable to a fully connected graph.

The membership grade $\mu(x)$ for any node or the link of the graph of a chain can determined by the following equation (1)

$$
\mu(x)=\frac{S+D}{N(N-1)}
$$

Where $S$ is the sum of degree of the nodes or links connected to the node or the link with the link under consideration, $D$ is the degree of the link or the node under consideration and $N$ is the number of nodes in the graph or links in the kinematic chain.

\section{ISOMORPHISM DEFINITION}

Two kinematic chains are said to be isomorphic if there is a one-to-one correspondence between the links of one chain to those of the other chain. If there is no such correspondence between the links of the two chains they are considered to be non-isomorphic. 


\section{TEST FOR ISOMORPHISM}

The similarity measure proposed by Wang [20] given by equation (2) and the similarity measure given by Pappis and Karacapilidis [21] given by equation (3) are used to compare the fuzzy sets A and B formed by using the membership grades of the nodes of the graphs of the two chains, if the value of the similarity measure comes out to be ' 1 ' the it can be concluded that there is a one to one correspondence between the graphs of the kinematic chains and they are said to be isomorphic.

$$
\begin{aligned}
& \operatorname{Sm}(A, B)=\frac{1}{N} \sum_{1}^{N} \frac{\min (\mu A(x), \mu B(y))}{\max (\mu A(x), \mu B(y))} \\
& \operatorname{Sm}(A, B)=\frac{\sum_{1}^{N} \min (\mu A(x), \mu B(y))}{\sum_{1}^{N} \max (\mu B(x), \mu B(y))}
\end{aligned}
$$

Where $\operatorname{Sm}(A, B)$ is the similarity measure between the fuzzy set $\mathrm{A}$ and $\mathrm{B}$

\section{TEST EXAMPLES}

Figure1-(a) shows a six link Watt's chain and its graph is shown in figure-1(b) along with a fully connected six node graph in figure1(c) now using the relation given by equation (1) it is found that for a fully connected or complete graph the membership grade comes out to be ' 1 ' for every node but for the graph of watt's chain the value of membership grade comes out to be between ' 0 ' and ' 1 ' calculated below

$$
\begin{gathered}
\mu A(1)=\frac{2+3+2+3}{6(6-1)}=\frac{10}{30}=0.3, \mu A(2)=\frac{7}{30}=0.23 \\
\mu A(3)=\frac{7}{30}=0.23, \mu A(4)=\frac{10}{30}=0.3, \mu A(5)=\frac{7}{30}=0.23 \\
\mu A(6)=\frac{7}{30}=0.23
\end{gathered}
$$

Fuzzy numbers obtained by the relation given by the equation (1) for the graph of the watt's chain are arranged in the decreasing order $[0.3,0.3,0.23,0.23,0.23$, and $0.23]$.Similarly the fuzzy numbers for the graph of Stephenson chain shown in figure-2(b) are obtained and arranged in decreasing order $[0.3,0.3,0.26,0.26,0.23,0.23]$

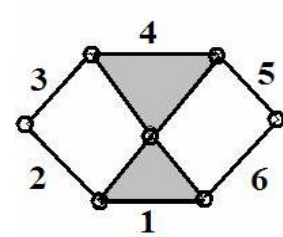

(a)

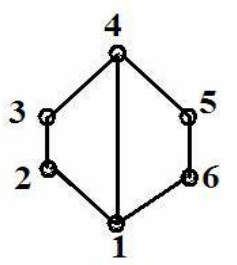

(b)

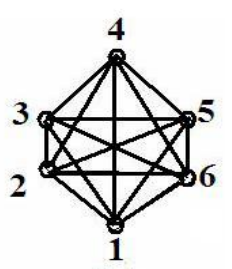

(c)
Fig: 1(a) Watt's chain (b) Graph of Watt's chain (c) 6-node complete graph

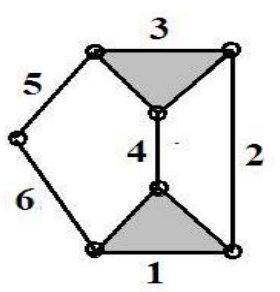

(a)

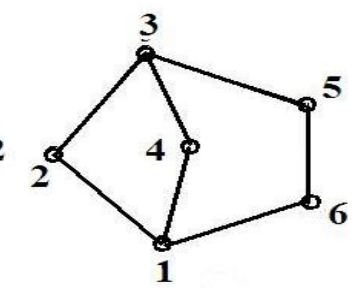

(b)
Fig: 2(a) Stephenson chain (b) Graph of Stephenson chain

Now the isomorphism among the two six link chain is checked with the help of the fuzzy numbers obtained by the relation given in equation (1) using the similarity measures proposed by Wang and Pappis. The value of similarity index using Wang's relation is 0.96 and by using Pappis relation is also 0.96 both the relation gives the value which is less than 1 which concludes that there is no one to one correspondence between the graphs of the two chains hence the two chains are nonisomorphic

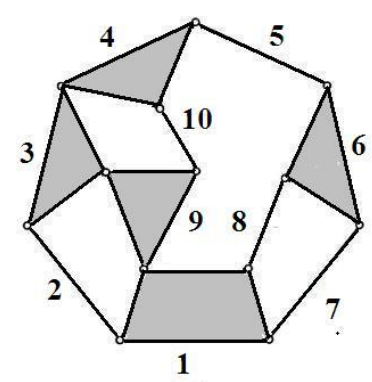

(a)

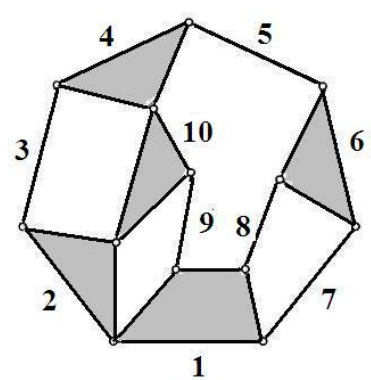

(b)
Fig: 3(a) \& (b) Ten link single degree of freedom chains

Two ten links single degree of freedom chains shown in fig3(a) and (b) available in the literature[13] were proved isomorphic by various researchers now to validate the proposed method the fuzzy numbers of the graphs of the two chains are used to show them isomorphic, the fuzzy numbers in decreasing order for the graphs shown in fig-4(a) \& (b) are $[0.13,0.12,0.11,0.11,0.1,0.1,0.1,0.1,0.08,0.08]$ and $[0.13,0.12,0$. $11,0.11,0.1,0.1,0.1,0.1,0.08,0.08]$ The value of similarity index using Wang's relation and Pappis relation comes out to be ' 1 ' which concludes that there is one to one correspondence between the graphs of the two chains hence the two chains are isomorphic.

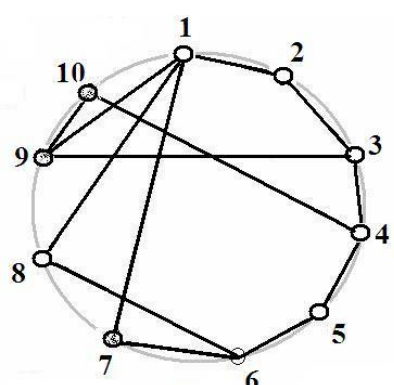

(a)

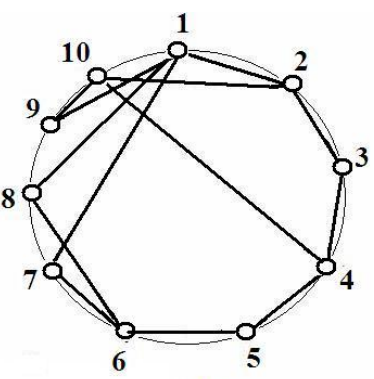

(b)
Fig: 4(a) \& (b) Graphs of the chains shown in fig-3(a) 


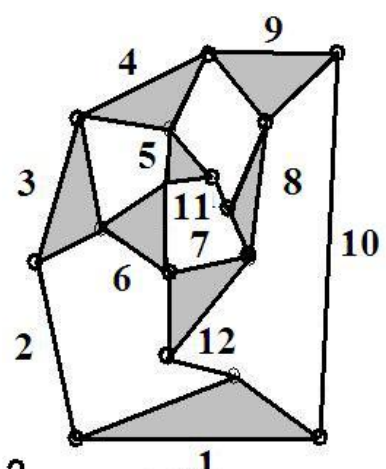

(a)

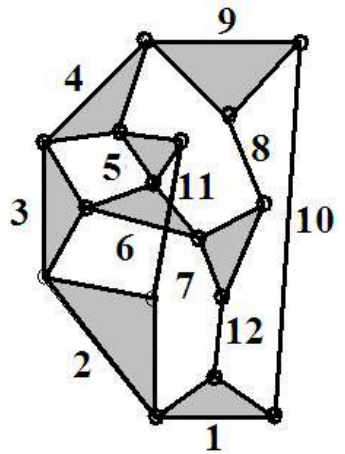

(b)
Fig: 5(a) \& (b) Twelve link chains both having single degree of freedom

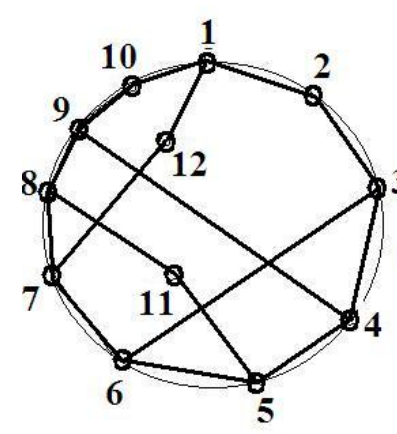

(a)

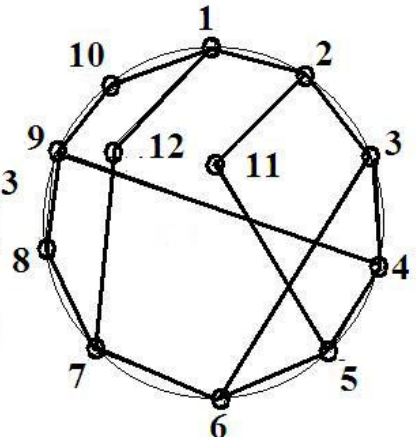

(b)
Fig: 6(a) \& (b) Graphs of the chains shown in the fig-5(a) \& (b)

Another example of two twelve link single degree of freedom chains shown in fig-5(a) \& ( b) which were proved nonisomorphic by Kong et al [13] and Ding [17] to validate the proposed method are used here. The fuzzy numbers obtained from the graphs of the two chains shown in fig-6(a) $\& 6$ (b) are arranged in decreasing order $[0.09,0.09,0.083$, $0.083,0.083,0.083,0.083,0.068,0.06,0.06,0.06,0.06]$ and $[0.09,0.09,0.09,0.083,0.083,0.075,0.075,0.075,0.06$, $0.06,0.06,0.06]$. The value of similarity index using Wang's relation is 0.968 and by using Pappis relation is 0.926 both the relation gives the value which is less than 1 which concludes that there is no one to one correspondence between the graphs of the two chains hence the two chains are nonisomorphic

\section{CONCLUSIONS}

The proposed method based on the theoretical analysis is capable of identifying isomorphism in planer kinematic chains of any size and even in cospectral graphs has been verified by several examples. It is the easiest and quick among the methods proposed so far and the reliability of this method is based on the fact it has been found to be successful in not only identifying all known 16 kinematic chains of 8 links, 230 kinematic chains of 10 links having 1-d.o.f and 40 kinematic chains of 9 links having 2-d.o.f but also on all the counter examples reported earlier literature. The inherent relation between the fuzzy numbers obtained for the kinematic chain graphs needs further study to identify the extent of parallelism between the input and the output links.

\section{REFERENCES}

[1] Davies T.H. and Crossley F.E. 1966. Structural analysis of plane linkages by Franke's condensed notation, J. Mech., Vol. 1(2), pp. 171-183.
[2] Yadav J.N. and Pratap. C.R. 1996. Computer aided detection of isomorphism among kinematic chains and mechanisms using the link-link multiplicity distance concept, Mech. Mach. Theory, Vol. 31(4), pp. 873-877.

[3] Mruthyunjaya T.S. and Balasubramanian H.R.1987. In quest of reliable and efficient computational test for detection of isomorphism in kinematic chains, Mech. Mach. Theory, Vol. 22(2), pp. 131-139.

[4] Rao A.C. and Prasad V.V.N. Raju. 2000. Loop based detection of isomorphism among chains, inversions and type of freedom in multi degree of freedom chain. J. Mech. Des., Vol. 122(1), pp. 31-41.

[5] Yan H.S. and Hwang W.M. 1983. A method for identification of planar linkage Chains. Mech. Tran. Auto. Des. ASME Trans., Vol. 105(4), pp. 658-662.

[6] Mruthyunjaya T.S. 1984. A computerized methodology for structural synthesis of kinematic chains: Part 1formulation. Mech. Mach. Theory, Vol. 19(6), pp. 487 495.

[7] Agarwal V.P. and Rao J.S. 1985. Identification of multiloop kinematic chains and their paths. J. Int. Eng. (I) ME, Vol. 66, pp. 6-11.

[8] Ambekar A.G. and Agarwal V.P. 1987. Canonical numbering of kinematic chains, mechanisms, path generators and function generators using min codes. Mech. Mach. Theory, Vol. 22(5), pp. 453-461.

[9] Rao A.C. and Varda. D. Raju 1991. Application of the hamming number technique to detect isomorphism among kinematic chains and inversions. Mech. Mach. Theory, Vol. 26(1), pp. 55-75.

[10] Shin J.K. and Murthy S. Krishna. 1994. On identification and conical numberings of pin jointed kinematic chains. J. Mech. Des ASME, Vol. 116(1), pp. 182-188.

[11] Hwang W.M. and Hwang Y.W... 1992. Computer aided structural synthesis of planar kinematic chains with simple joints. Mech. Mach. Theory, Vol. 27(2), pp. 189199.

[12] Yadav J.N., Pratap C.R. and Agarwal V.P.1996. Computer aided detection of isomorphism among kinematic chains and mechanisms using the link-link multiplicity distance concept. Mech. Mach. Theory, Vol. 31(7), pp. 873-877.

[13] Kong F.G., Q. Li and Zhang W.J. 1999. An artificial neural network approach to mechanism kinematic chain isomorphism identification. Mech. Mach. Theory, Vol. 34(2), pp. 271-283.

[14] Quist F.F. and Soni. A.H. 1971. Structural synthesis and analysis of kinematic chains using path matrices. In. Proceedings of the 3rd World Congress for Theory of Machines and Mechanisms, pp. D161-D176.

[15] Chang Z., Zhang C., Yang Y. and Wang Y. 2002. A new method to mechanism kinematic chain isomorphism Identification. Mech. Mach. Theory, Vol. 37(4), pp. 411417.

[16] Sunkari R.P. and Schmidt L.C. 2006. Reliability and efficiency of the existing spectral methods for isomorphism detection. J. Mech. Des., Vol. 128(6), pp. 1246-1252. 
[17] Ding H. and Huang Z. 2007. The establishment of the canonical perimeter topological graph of kinematic chains and isomorphism identification. J. Mech. Des., Vol. 129(9), pp. 915-923.

[18] Hasan A. and Khan R.A. 2008. Isomorphism and inversions of kinematic chains up to ten links using degrees of freedom of kinematic pairs. Int. J. Comp. Methods, Vol. 5(2), pp. 329-339.
[19] Rao. A.C. 2000. Application of fuzzy logic for the study of isomorphism, inversions, symmetry, parallelism and mobility in kinematic chains. Mechanism and Machine Theory 35 pp.1103-1116

[20] Wang WJ. 1997. New similarity measures on fuzzy sets and on elements. Fuzzy Sets Syst.85:305-309.

[21] Pappies CP, Karacapilidis NI.1993. A comparative assessment of measures of similarity of fuzzy values. Fuzzy Sets Syst.56:171-174. 